\title{
ADMET PREDICTORS ARE THE TOOLS FOR THE ENHANCEMENT OF DRUG DESIGN AND DEVELOPMENT: A SYSTEMATIC REVIEW
}

\author{
M. Manoj Kumar*, B. Renuka Swathi' ${ }^{1}$, Shashank Gorityala², G. Padma Sree1 \\ *Department of Pharmacology, Sir C.R.Reddy College of Pharmaceutical Sciences, Eluru, AP, India. \\ ${ }^{1}$ Department of Pharm D, Sir C.R.Reddy College of Pharmaceutical Sciences, Eluru, AP, India. \\ ${ }^{2}$ Department of Bioanalytical Chemistry, Covance Laboratories, Madison, WI 53704, USA. \\ *Corresponding author e-mail: manoj.maguluru@gmail.com
}

\begin{abstract}
:
Computational or computer aided drug design is method of using computational perspectives in identifying, analyzing, developing and discovery of drugs and related biologically active compounds. Though the thought of computational drug discovery was started in 1970s, it was rather slow in early years but it played an important role in discovery of drugs in past three decades. Now it was thought to be an answer for industrial burdens in drug discovery. Conventional drug discovery is a long and expensive process. It may take years to decades before releasing the drug in to the market. If a new candidate fails in ADME properties or if it produces abnormal toxicity in humans, we cannot release the drug into the market. The time and money spent on its development turns waste. Due to this, pharmaceutical industry suffers huge burden. To avoid this late stage attrition we can make use of computational tools. The ADMET predictor tools can be better used to predict the properties of the drug in early and therefore time and money can be saved. This article reviews different computational tools used frequently in prediction of ADMET properties of new entities.
\end{abstract}

Key words: Ligand based drug design, ADMET predictors, Pharmacokinetic prediction tools, Prediction of molecular properties.

\section{INTRODUCTION:}

Disease or disorder is a pathological condition where the physiology of the human body is deviated from the normal either due to malfunctioning of one or some particular proteins or due to invasion of microorganisms. Drugs used in the management of disorder are aimed to correct the deviation resulted in the physiology of enzymes or any other proteins. In case of diseases, drugs aim to kill or stop the growth of microorganisms. Inappropriate use of drugs in the treatment of diseases may result in development of resistance to those drugs which are being used; where as in case of disorders, prolonged use of medicines may result in development of tolerance by humans to those drugs. In both the cases further use of those drugs may not give good therapeutic effect. An alternate drug is required for better treatment. This necessitates the invention of new drugs.

New drug design and development by conventional method is a hectic task. It consumes lots of time and money. After spending all the time and money, if a new drug candidate fails to produce desired therapeutic action; it is total waste. This causes huge late stage attrition on pharmaceutical industry in the development of new drugs. Prior prediction of drug 
properties and their possible therapeutic effects may helpful in avoiding this loss. In silico methods of drug design finds use in this area. Computational methods can predict the fitfulness of the new drug entity at the active site of required target (receptor) and also helpful for prediction of ADMET properties of new drug molecule.

Computational drug design is of two types. Structure based drug design (SBDD) and ligand based drug design (LBDD). In SBDD, the ability and affinity of a ligand to bind to the active site of the biological macromolecular target is estimated. This can be carried out when the three dimensional structure of target protein is known. LBDD is used while we lack the 3D information of a target (receptor). It purely depends on the information of the existing ligands which are able to bind to the target of interest. It is useful in lead identification, lead optimization and prediction of ADMET properties of a new entity in drug design. This article is aimed to review the various ligand based drug design tools available which decrease the late stage attrition on pharmaceutical industry by predicting ADMET properties of a new entity.

\section{DSSTox [1][2]}

In 2004 US EPA (Environmental Protection Agency) launched a database called DSSTox(Distributed Structured Searchable Toxicity). DSSTox focus on resolving chemical errors and conflicts. Its goal is to assign accurate chemical structure data. Accurate chemical structure data is a very important input to structure based predictive models that are supporting the hazard and risk assessments. DSSTox aims to establish the relation of ligand's chemical structure data with the existing data on toxicity and promotes the structure based data with chemistry based structure that extends the regulation of toxicology.
The DSSTox contains these three features:

- It adopts and encourages the standard file format use for public toxicity database which includes chemical structures which are simply imported into accessible chemical relational database applications.

- Implements the approach of a scattered source which will allow decentralized and free public access to toxicity data sources.

- The DSSTox network is increased as it is useful for public, commercial, academic, industrial groups.

\section{Topkat [3]}

Topkat is based on QSAR system. It is developed by Accelrys Inc. This predicts wide range of toxicity end points that includes mutagenicity, developmental toxicity, and rodent carcinogenicity.

Topkat is derived by using 2D molecules, electronic and spatial descriptors. Validation method applied in Topkat for prediction is OPS (Optimal Predictive Space). Any predictions outside the OPS are not supported by the model and exhibit deviation from the experimental values. The validation criteria must be satisfied for reliability of prediction.

\section{CPDB (Carcinogenic Potency Database) [4]}

It is a systematic resource that analyses the outcome of chronic animal cancer tests performed on chemicals. The analysis provides sufficient information about carcinogenesis. It reports information on positive and negative experiments both qualitatively and quantitatively. For evaluating carcinogenic activity and potency a set of rules are designed. The experimental results are standardized as an accessible resource which is useful for analysis of research and regulatory issues in carcinoma. 


\section{PreADMET predictor [5]}

This is a web based method which predicts drug likeliness and ADMET properties. PreADMET predictor can calculate molecule descriptors more than 900. It includes topological, electrostatic, constitutional, physicochemical and geometric descriptor for the prediction of ADME properties. PreADMET collects information regarding ADME and toxicity to regulate physiologically based pharmacokinetic model. It calculates toxicity based on Ames mutagenicity test. The values of prediction are positive or negative. Based on NTP (National Toxicological Program) and US FDA carcinogenicity is predicted.

\section{Molinspiration[6]}

Molinspiration is a Java based software which can implement in any operating system such as Windows, Mac, UNIX. Molinspiration helps to find out Log P, polar surface area, hydrogen bonding and other sub atomic properties. It also provides bio activity score for G-protein coupled receptor ligands, kinase inhibitors, particle channel modulators, atomic receptors. It provides ten Cheminformatics softwares, such as Mib engine, Misearch engine, Miscreen engine, Galaxy, molinspirationclusterer, molinspiration property calculator, molinspiration molecule viewer, molinspiration data viewer, molinspiration depiction, database of bio active substitutes and linkers.

\section{ADMET predictor [7][8]}

ADMET predictor is a software tool available in simulations plus. It is designed using artificial neutral network ensemble (ANNE) models. It is trained with clear description of drugs and it is selected for its higher ability in analysis of prediction and sensitivity. It estimates various pharmacokinetic parameters such as lop $\mathrm{P}, \log \mathrm{D}$, hydrogen bonding, polar surface zone, human intestinal www.ijapbjournal.com absorption, plasma protein binding,metabolism sites. ADMET predictor creates high quality QSAR/QSPR models rapidly and easily based on the data. Before invoking the ADMET predictor polyatomic counter ions must be removed.

\section{Moka[9][10]}

It is one of the new software useful for prediction of organic compounds pka value. It is based on QSPR approach. The training set contains 26,000 pka values. By using GRID field physico chemical properties compound is encoded. Descriptors from GRID field provide fast and accurate calculations. These descriptors also encode 3 dimensional features of a chemical structure to identify hydrogen bonding and steric effects. Key features are it is accurate, fast, automatic and self-trainable.

\section{SHOP (Scaffold HOPing) [11][12][13]}

It was discovered by schneider et al. and he defined the Scaffold hoping as "Identification of iso-functional molecular structures with significantly different molecular backbones" SHOP methodology identifies new scaffolds by analyzing the 3 dimensional structure similarity to that of query Scaffold. The main aim is to discover the substitutes of query scaffold (new discovery drug) without disturbing configuration and interaction pattern of a central fragment. In a fully integrated approach, it maintains 3D interaction capabilities and at the same time, the synthetic feasibility and ADME profiles of the potential compounds.

\section{Tsar [14][15]}

Tsar is a fully integrated QSAR. It is used for determination of drug design and lead optimization. Tsar can be used throughout the drug discovery process that is from selection of leads to final screening. 
Applications:

- Exploring physical and chemical properties to understand that which property promotes drug activity.

- Helps in lead selection.

- It develops the predictive models of activity.

- It can be used to model fully flexible docking.

- Used to predict Pka values.

- It helps in identifying new compounds with automatic ADME prediction.

- This softwar computes Lipinski's rule for identifying drug like compounds. (Lipinski's rule: It helps to determine if biologically active compounds are likely to have the physico-chemical properties to be orally bioavailable).

- This software also features a formal modeling that automatically shows researchers a tree chart, that chart presents ranks to drug leads in order of importance.

- It is easy to use and it is equally accessed by medicinal and computational chemists.

Advantages: Accelerates drug design. Promotes the selection of single compounds for screening. Helps in eliminating undesirable properties in lead compounds.

\section{Volsurf[16]}

Generally 3D molecular fields contain large data which may not be relevant to problem. So, novel tools like Volsurf are required. The main concept of Volsurf is to collect the data from 3 dimensional images and to link the experimental information with molecular structures for easy understanding and interpretation.

The individuality of volsurf remains mainly in surfaces, volume and other related descriptions that are easily acquired from 3 dimensional fields with computational algorithms. Volsurf descriptors are easily acquired for small, medium, large molecules and biopolymers that include DNA, proteins and peptides which is the main advantage of this software.

Key features: According to experimental information, ADME descriptors are calculated and statistical modelling is performed. New compound behavior is predicted based on ADME models

\section{OncoLogic}

It was developed by United States Environmental Protection Agency in cooperation with Logichem. OncoLogic expert system assesses the potential of chemicals that cause cancer. It finds out the carcinogenicity by applying the structure activity relationship analysis rules and incorporating the mechanisms of action and epidemiological studies. It also contains toxicological information relay to carcinogenicity assessment. The cancer expert system is composed of four subsystems that assess fibres, metals, polymers and organic chemicals of various chemical structures. The user requires a limited knowledge to choose the suitable subsystems.

\section{Lazar [17]}

It is open source software that predicts toxicological endpoints like mutagenicity and carcinogenicity, human liver toxicity by examining the structural fragments. It is based on statistical algorithms. Lazar analyses similarities of chemicals as toxicity dependent's values but not as absolute values. Lazar provides confidence index and it performs automatic applicability domain for each prediction. It can be used without expert's knowledge.

Lazar mutagenicity predictions are depends on k-NN algorithm and two datasets. Two datasets are kazius or Bursi and Benchmark 
dataset. Prediction confidence is between 0 and 1. Prediction confidence gives an idea about absence or presence of compounds within the applicability domain. Confidence value more than 0.025 is the cut off for compounds with applicability domain. The accuracy of prediction decreases with the confidence value.

\section{ToxBoxes[18]}

Now ToxBoxes are called as ACD/ToxSuite. It is developed by ACD/labs and Pharma Algorithms. It gives prediction about many toxicity endpoints. It includes various endpoints such as Genotoxicity, ER binding affinity, hERG inhibition, CYP3a4 inhibition and organ specific health effects. Predictions are provided as confidence intervals. The software identifies and visualizes the specific structural toxicophore. It gives insight about the molecular parts that are responsible for toxicity.

\section{PASS [19[20]}

PASS means prediction of activity spectra of substances.

It is a QSAR modeling program. Institute of Biomedical Chemistry developed first version of PASS in Russian academy of medical sciences. PASS is available in GeneXplain. PASS predicts the biological activities of pharmaceutical compound of a lead. It is formed on the basis of comparison of existing structures of library.

PASS prediction accuracy is 95\%. Biological activities predicted in PASS are described quantitatively in terms of active/inactive or yes/no. To know the biological activities new chemical structure is converted into 2D structural formula. MNA descriptor represents molecular structure in PASS. If substances are having same MNA descriptors then they are said to be identical in PASS.

Uses: It provides information about mechanism of action and new effects for www.ijapbjournal.com known compounds in personal database and corporate databases. It selects the most favourable substances from the samples for screening. It finds new leads for the given biological activity profiles.

\section{DEREK for Windows [21]}

It is a knowledge based system for the toxicity prediction. It is developed by Lhasa Ltd. It is derived from the DEREK system and the StAR project.

Derek for windows contains examples, alerts, and rules.This program has a user friendly interconnection that allows the query structures either in ISIS/Draw or molfiles or sketch files.

Derek for windows contains 50 alerts that cover a wide variety of toxicity end points in bacteria, mammals, humans.

A toxicophore in alert is associated with comments, examples and literature references. The important characteristic of Derek for windows is clear reporting of the reason under each prediction. Derek for windows rules are based either on mechanism of action of chemicals or on empirical relation. Rules are developed based on the information collected from the data published and toxicological expert's suggestions. Prediction of toxicity is the result of these processes. The process first find outs whether any of the alter matches with the toxicophore. The reasoning engine then identifies the structure toxicity. There are nine confidence levels they are certain, probable, plausible, equivocal, doubted, improbable, impossible, open, and contradicted.

\section{Swiss ADME [22]}

Swiss ADME is a web based ADME predictor tool which is available freely. It can be used by the persons who are not familiar with CADD and prediction software. It is a user friendly tool. When compared to the other

IJAPB 
web based tools for the prediction of ADME or Pharmacokinetics it has advantages like accepting different kinds of input, possibility of computing data of multiple molecules, and the chance to present, save and share the results molecule wise individually or may present result through globally accepted graphical representation. SwissADME is integrated in the SwissDrugDesign workspace.

\section{pkCSM [23]}

In the field of drug design and development pkCSM is an approach to develop predictive models for ADMET properties in drug design and development using graph based signatures. It is available to the users through, http://structure. bioc.cam.ac.uk/ pkcsm, a free web server. It was supposed to be better method than the other by discoverers. It rapidly evaluates ADMET properties of drug molecules in its integrated platform. It can handle chemically diverse and challenging datasets. It can handle large datasets as well. The webserver was designed in such a manner that it does not retain any matter after use. Hopefully this protects the secrecy of the projects carried out by researchers. This is comparatively easier method to handle.

\section{admetSAR}

admetSAR is an open source database for structure activity relationship of molecules. This database continuously collects, curate and manages ADMET and related properties from the latest literature. You can make a query in the database using its user friendly interface through either the chemical name, common name, CAS registry number or through chemical structure of a molecule. The database had been provided with 22 qualitative classification models and 5 quantitative regression models which possess high accuracy in predicting the ADMET properties novel drugs.

\section{CONCLUSION}

Predictors used in computer-based method serve as useful tools for the early prediction of ADME and Toxicity properties of the new entities. Most of the tools discussed are used in the prediction of ADME properties and some are used to predict toxicity of the new candidates. Some tools like CPDB and OncoLogic predict carcinogenicity of the drugs. DSSTox is based on scattered source approach and helpful for assessment of tocxicity. Topikat is based on QSAR system and used for the prediction of wide range of toxicity including mutagenicity and carcinogenicity. CPDB, Carcinogenic potency Database, and OncoLogic analyses and provides sufficient information on ability of carcinogenesis of drugs. preADMET predictor is based on over 900 descriptors like topological, electrostatic, constitutional, physicochemical and geometric properties and useful prediction of ADMET properties of new entities. Moka-pka model is useful for estimation of pka values of organic compunds. ADMET predictor is designed using artificial neural networks (ANN) and has high ability in analysis of prediction and sensitivity of predictiong ADMET properties of drugs. Tsar is fully integrated QSAR model which is useful in total process of drug discovery, from the stage of lead optimization to ADMET Assessment and final biological screening. Each method has its specific advantages and some limitations, and all can contribute to rational drug design.Some of them are freely available to use readily by researchers.

\section{REFERENCES}

[1] Umer Rashid, Syed Fahad Hassan, Samina Nazir, Abdul Wadood, Muhammad 
Waseem, Farzana Latif Ansari, Synthesis, docking studies, and in silico ADMET predictions of some new derivatives of pyrimidine as potential KSP inhibitors, Med Chem Res,2014,24;1-12.

[2] Ann M. Richarda,* and ClarLynda R. Williamsa,b, Distributed StructureSearchable Toxicity (DSSTox) Public Database Network: A Proposal, Mutation Research,2002,499(1):1-26.

[3] Andrew Worth, Silvia Lapenna, Elena Lo Piparo, Aleksandra Mostrag-Szlichtyng and RositsaSerafimova, The Applicability of Software Tools for Genotoxicity and Carcinogenicity Prediction: Case Studies relevant to the Assessment of Pesticides, Joint Research Centre - Institute for Health and Consumer Protection,2010,75 pages.

[4] Lois Swirsky Gold, , †,1 Neela B. Manley, $\dagger$ Thomas H. Slone,* Lars Rohrbach, $\neq$ and Georganne Backman Garfinkel†, Supplement to the Carcinogenic Potency Database (CPDB): Results of Animal Bioassays Published in the General Literature through 1997 and by the National Toxicology Program in 19971998, TOXICOLOGICAL SCIENCES, 2005, 85;747-808.

[5] Yeni*, Supandi, FajarMerdekawati, In silico toxicity prediction of 1-phenyl-1(quinazolin-4-yl) ethanol compounds by using Toxtree, pkCSM and preADMET, Pharmaciana, Vol.8, No.2, Nov 2018, Page. 205-216.

[6] Ajeet Singh, Shafaque Zahra, and Shailesh Kumar, In-silico Tools in Phytochemical Research, Phytochemistry: An in-silico and in-vitro Update, National Institute of Plant Genome Research,chapter 19;2019,351-372.
[7] Rodolfo GonellaDiaza; Serena Manganelli; Antonella Esposito; Alessandra Roncaglioni; Alberto Manganaro; Emilio Benfenati., Comparison of in silico tools for the evaluation of rat oral acute toxicity, SAR QSAR Environ Res. 2015;26(1):1-27.

[8] Umer Rashid, Syed Fahad Hassan, Samina Nazir, Abdul Wadood, Muhammad Waseem, Farzana Latif Ansari, Synthesis, docking studies, and in silico ADMET predictions of some new derivatives of pyrimidine as potential KSP inhibitors, Med Chem Res,2014,24;1-12.

[9] Rudiger Klein, Knowledge Modeling In Design Framework, J. S. Gero (ed.),2000,7102.

[10] Gabriele Cruciani*a), Francesca Millettia), LorianoStorchib), GianlucaSfornab), and Laura Goraccib), In silico pKa Prediction and ADME Profiling, CHEMISTRY \& BIODIVERSITY,2009,vol.6;1812-1821.

[11] Rikke Bergmann,, , Anna Linusson, $\neq, \S$ and Ismael Zamora|, $\perp$, SHOP: Scaffold HOPping by GRID-Based Similarity Searches, J. Med. Chem. 2007, 50, 27082717.

[12] Fabien Fontaine,[b] Simon Cross,[c] GuillemPlasencia,[b] Manuel Pastor,[a] and Ismael Zamora*[a], SHOP: A Method For Structure-Based Fragment and Scaffold Hopping, ChemMedChem 2009, 4, 427 - 439 .

[13] Rikke Bergmann, ${ }^{*}, \dagger$ Tommy Liljefors, $\uparrow$ Morten D. Sørensen, $\neq$ and Ismael Zamora§,|, SHOP: Receptor-Based Scaffold HOPping by GRID-Based Similarity Searches, J. Chem. Inf. Model. 2009, 49, 658-669.

[14] Oleg V. Stroganov,1,2Fedor N. Novikov,1 Alexey A. Zeifman,2 Viktor S. Stroylov,1 and Ghermes G. Chilov1,2*, TSAR, a new

IJAPB 
graph-theoretical approach to computational modeling of protein sidechain flexibility: Modeling of ionization properties of proteins, Proteins 2011; 79:2693-2710.

[15] Pooja Mishra1, Vijay Tripathi1, Brijesh Singh Yadav2*, Insilco QSAR modeling and drug development process, GERF Bulletin of Biosciences, December 2010, 1(1): 37-40.

[16] Gabriele Cruciania ,*, Manuel Pastora, Wolfgang Gubab, VolSurf: a new tool for the pharmacokinetic optimization of lead compounds, European Journal of Pharmaceutical Sciences,2000,11 Suppl. 2; S29-S39.

[17] Andreas Maunz,Martin Gütlein, Micha Rautenberg, David Vorgrimmler,Denis Gebele, and Christoph Helma. lazar: a modular predictive toxicology framework. Frontiers in Pharmacology, 4:38. doi:10.3389/fphar.2013.00038.

[18] MojcaFuartGatnik and Andrew Worth, Review of Software Tools for Toxicity Prediction, Joint Research Centre Institute for Health and Consumer Protection,2010,13 pages.

[19] Megan L Peach1, Alexey V Zakharov2, Ruifeng Liu3, Angelo Pugliese2,4, Gregory Tawa3, Anders Wallqvist3\& Marc C Nicklaus*2, Computational tools and resources for metabolism-related property predictions. 1. Overview of publicly available (free and commercial) databases and software, Future Med. Chem. (2012) 4(15), 1907-1932.
[20] Prasad G. Jamkhandea,?], Mahavir H. Ghantea, Balaji R. Ajgundeb, Software based approaches for drug designing and development: A systematic review on commonly used software and its applications, Bulletin of Faculty of Pharmacy, Cairo University,2017,55; 203-210.

[21] Carol A. Marchant, Katharine A. Briggs, and Anthony Long, In Silico Tools for Sharing Data and Knowledge on Toxicity and Metabolism: Derek for Windows, Meteor, and Vitic, Toxicology Mechanisms and Methods,2008,18:177-187.

[22] Antoine Daina, OlivierMichielin, and Vincent Zoete.SwissADME: a free web tool to evaluate pharmacokinetics, druglikeness and medicinal chemistry friendliness of small molecules.Scientific Reports, 7(1). doi:10.1038/srep42717.

[23] Douglas E. V. Pires, Tom L. Blundell, David B. Ascher. pkCSM: predicting smallmolecule pharmacokinetic properties using graph-based signatures. Journal of Medicinal Chemistry, 58 (9), p. 40664072, 2015.

[24] Cheng, F., Li, W., Zhou, Y., Shen, J., Wu, Z., Liu, G., ... Tang, Y. (2012). admetSAR: A Comprehensive Source and Free Tool for Assessment of Chemical ADMET Properties. Journal of Chemical Information and Modeling, 52(11), 30993105.

\section{How to cite this article:}

Manoj Kumar et al., ADMET Predictors Are the Tools for the Enhancement of Drug Design and Development: A Systematic Review. Int. J. Adv. Pharm. Biotech., 2018; 4(4): 6-13. 